\title{
Does gradients or swim up procedure produce baby of desired gender?
}

\begin{abstract}
Background: Pre selection of baby gender is still desire of many parents. A number of procedures have been used including $\mathrm{X}$ and $\mathrm{Y}$ sperm separation by various semen processing procedures for intrauterine insemination (IUI), sperm sorting by flowcytometry, fluorescent in situ hybridization and preimplantation genetic testing (PGT). Due to low cost, simplicity and easy availability, various X-and Y-bearing sperm separation procedures followed by IUI have been commonly used but with conflicting accuracy. The most common sperm separation procedures include; gradients filtration, swim up, swim down and albumin gradients filtration. The objective of this study was to investigate whether swim-up or density gradient semen processing followed by IUI results in significant deviation in the ratio of male and female babies.
\end{abstract}

Methods: The study was carried out retrospectively at Thuriah Medical Center. The data of 990 IUI cycles from Jan 2016 to Dec 2017 were analyzed. The semen samples were processed by gradient filtration, swim up or simple wash depending on quality of the sample and desire of patient. Data on female and male age, total progressively motile sperm inseminated, pregnancy rate and delivery of male or female baby were recorded and analyzed.

Results: Gradient filtration, swim up and simple wash were used in 69, 26 and $5 \%$ samples, respectively. Male or female ages in different groups were not significantly different $(\mathrm{P}<0.05)$. Biochemical pregnancy rates were highest in the swim up group and lowest in the simple wash group.

Conclusion: Based on the results of this study, the gender ratio did not change significantly from the natural ratio of about 50/50; therefore, sperm separation by these procedures for gender selection is not accurate.

Keywords: IUI, gender selection, gradients filtration, swim up, simple wash, abortion, PGT

\section{Introduction}

Human desire to pre select baby gender has a long history and possibly existed in all cultures. ${ }^{1}$ The reasons include; cultural, social and economic preferences, gene linked diseases and family balancing. ${ }^{2}$ Even the infertile patients have significant demand for preimplantation sex selection, with a significant portion of this demand coming from patients who do not have any child or have all children of same sex. ${ }^{3}$

The publication of article on separation of X- and Y- bearing spermatozoa using albumin gradients ${ }^{4}$ gained high popularity. Later, another study ${ }^{5}$ suggested the possibility that the swim-up technique may help preselect male offspring. The density gradient procedure is reported to concentrate X-bearing sperm in the sediment. Kaneko et al $1983^{6}$ found that after centrifugation, the content of Y-bearing sperm in the fraction lighter than $1.06 \mathrm{gm} / \mathrm{ml}$ was $73.1 \% \pm 3.3 \%$ and was reduced gradually along the density increment and it was $27.4 \% \pm 3.4 \%$ in the sediment (more than $1.11 \mathrm{gm} / \mathrm{ml}$ ).

Several studies have been carried out to select desired gender of baby with conflicting results. Non-significant influence was found on the mean ratio of $\mathrm{X}$ - to Y-bearing spermatozoa after semen was prepared with Percoll gradients or after swim up as compared to that in fresh semen. ${ }^{7,8}$ Before the advent of pre-implantation genetic testing (PGT), the most common laboratory procedures used for sperm separation were gradient filtration, swim up or swim down, albumin gradient filtration and sperm sorting by flow cytometry followed by IUI or ICSI. However, due to high cost of flow cytometry technique and PGT, most laboratories use either gradient or swim up procedure.
Volume 10 Issue I - 2019

\author{
Murid Javed, Ruaa Laqwer, Bilal Mahouk, \\ Abdulla Kannachath, Suleiman Najashi, \\ Hamad Sufyan \\ IVF Laboratory, Thuriah Medical Center, Saudi Arabia
}

Correspondence: Murid Javed, PhD, IVF Laboratory Director, Thuriah Medical Center, 244Makkah Road, Riyadh, I I523, Saudi Arabia,Tel 96654 9146434, Email murid.javed@thuriah.com.sa

Received: January 25, 2019 | Published: February 14, 2019
The underlying concept is that swim-up technique enriches Y-bearing sperm and density-gradient separation enriches X- bearing sperm. Among the sperm separation techniques, sperm sorting by flow cytometry separated $\mathrm{X}$ and $\mathrm{Y}$ bearing sperm deviating significantly from the natural ratio of 50:50 but not $100 \%$. The actual outcome is measured based on the delivery of desired gender as fertilization may happen by any sperm $\mathrm{X}$ or $\mathrm{Y}$ if the separation techniques is not separating at $100 \%$ level. The objectives of this study were to investigate whether swim-up; density gradient and simple wash followed by IUI result in significant deviation in the ratio of male and female babies.

\section{Materials and methods}

The data were collected from 990 couples undergoing IUI cycles at Thuriah Medical Center from January 2016 to December 2017. The semen was collected on site after 3-5 days abstinence and processed by gradient filtration, swim up or simple wash (Figure 1) depending on the quality of sample and gender preselection desire of patient.

The gradients were prepared in $15 \mathrm{~mL}$ Falcon conical tube (Fisher Scientific); $1.5 \mathrm{~mL}$ of $80 \%$ gradient (V-Grad 80, VITROMED, Germany) at the bottom and $1.5 \mathrm{~mL}$ of $40 \%$ gradient (V-Grad 40 , VITROMED, Germany) on the top of it. The semen sample was gently layered on the top of the $40 \%$ gradient. The tube was centrifuged at $1500 \mathrm{rpm}$ for 20 minutes. The pellet was washed in $4 \mathrm{~mL}$ V-Sperm Wash (VITROMED, Germany) by centrifugation at 1500rpm for 8 minutes. The final pellet was re-suspended in $0.4 \mathrm{~mL}$ V-Sperm Wash for IUI. 


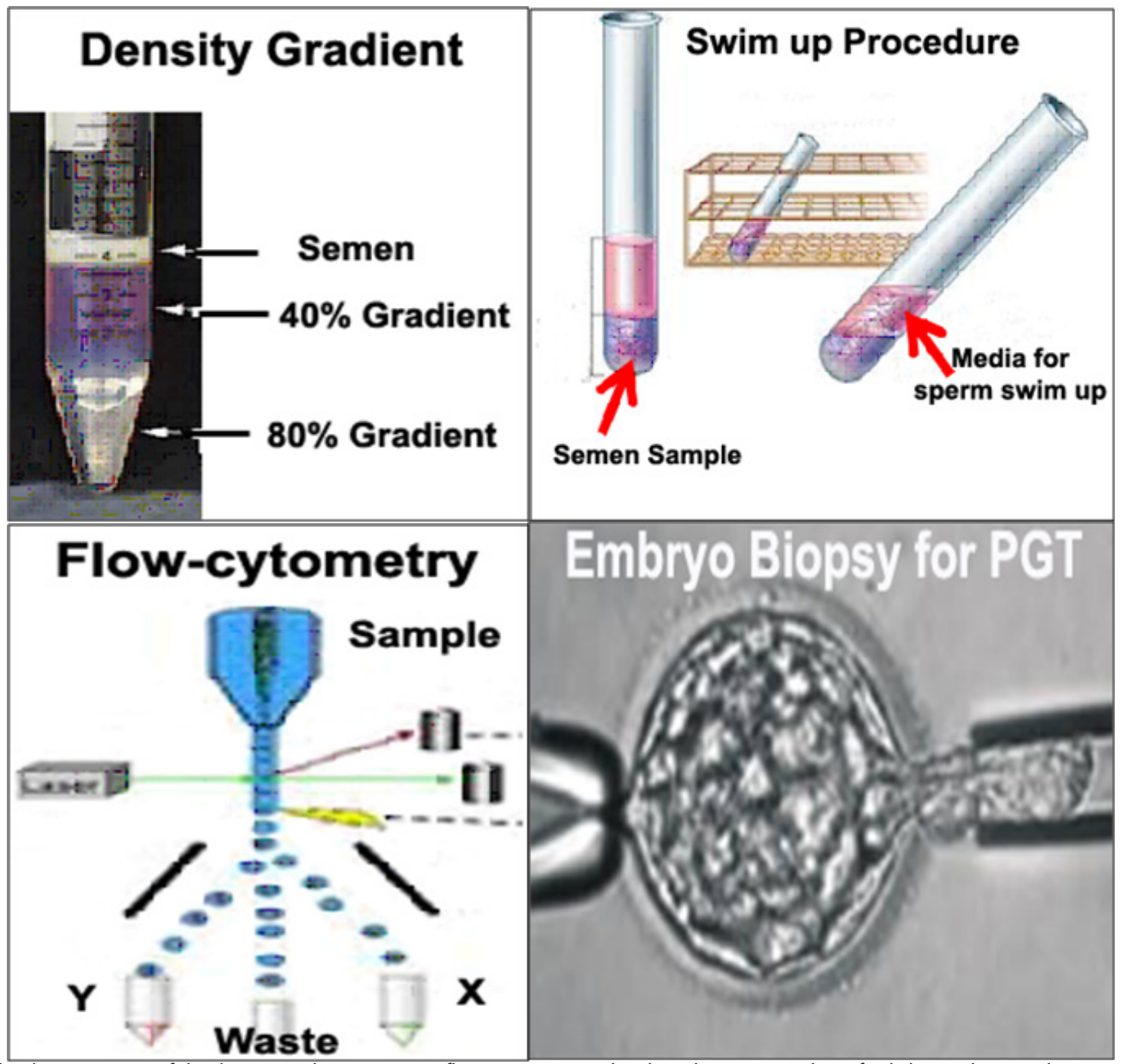

Figure I A graphical presentation of the density gradients, swim up, flow-cytometry and embryo biopsy procedures for baby gender preselection.

For the swim up procedure, each semen sample was mixed with equal amount of V-Sperm Wash and gently mixed in a $15 \mathrm{~mL}$ conical tube. The tube was centrifuged at 1500 rpm for 10 minutes. The supernatant was removed; the pellet was gently layered with 3 $\mathrm{mL}$ V-Sperm Wash and kept in incubator at $37^{\circ} \mathrm{C}$ for 30 minutes at 45 degree angle. The supernatant was gently taken out (leaving the pellet undisturbed) into a new tube and centrifuged at 1500rpm for 8 minutes. The pellet was then re-suspended in $0.4 \mathrm{~mL}$ for IUI.

The simple wash procedure was used for samples very low in sperm concentration. The semen sample was mixed with equal amount of V-Sperm Wash and centrifuged at 1500rpm for 10 minutes. The supernatant was removed and the pellet was re-suspended in $0.4 \mathrm{~mL}$ V-Sperm Wash for insemination.

The female partners were prepared for IUI procedure per standard protocol. Data on female and male age, total motile sperm inseminated, pregnancy rate and delivery of male or female baby were recorded and analyzed. The data are presented as Mean $\pm \mathrm{SD}$ and compared using student $t$-test for level of significance at 0.05 .

\section{Results}

The gradients filtration, swim up and simple wash procedures were used in $69 \% ; 26 \%$ and $5 \%$ samples, respectively. The male or female ages in different groups were not significantly different $(\mathrm{P}<0.05)$. All semen samples for the gradient and swim up procedures had final total progressively motile sperm concentration more than the $5 \times 10^{6}$ sperm, generally recommended for IUI. The simple wash procedure was used only for samples with poor sperm concentration and motility. In this group, the average total progressively motile sperm concentration was $4.5 \pm 1.5$ million for IUI (Table 1 ).

The number of procedures performed for each group, number of biochemically pregnant and the biochemical pregnancy rates are shown in Table 2. The swim up procedure achieved the highest pregnancy rate and the simple wash the lowest $(<0.05)$. The difference between gradient and swim up biochemical pregnancy rate was not statically significant. The overall biochemical pregnancy rate achieved was $17 \%$.

The data on clinical pregnancy rates and gender of the baby born in different procedures are shown in Table 3. Although higher clinical pregnancy rate was achieved when semen samples were processed by swim up, however, this difference was not statistically significant $(\mathrm{P}<0.05)$. In the density gradient group, which was expected to skew the gender ratio to more female born, the male and female ratio was 48/52. The swim up group which was expected to skew the gender ratio to more male born, the gender ratio was $50 / 50$. Therefore, in any of the procedures, the gender ratio did not significantly deviate from the natural ratio of about 50:50. 
Table I Different types of procedures, patient's ages and total motile sperm inseminated

\begin{tabular}{llllll}
\hline \multirow{2}{*}{ Type of procedure } & No. & Percent & Male age & Female age & Total progress. Motile sperm \\
\cline { 4 - 6 } & & & Mean \pm SD & Mean \pm SD & Mean \pm SD \\
\hline Gradient Procedure & 684 & 69.1 & $35 \pm 6.7$ & $30.3 \pm 5.2$ & $19.7 \pm 17$ \\
Swim-up Procedure & 261 & 26.4 & $37 \pm 7.4$ & $33.2 \pm 5.8$ & $25.6 \pm 16.8$ \\
Simple Wash & 45 & 4.5 & $33 \pm 2.8$ & $29.5 \pm 4.2$ & $4.5 \pm 1.5$ \\
Total Procedures & 990 & 100 & 35.1 & 31 & 16.6 \\
\hline
\end{tabular}

Table 2 Biochemical pregnancy rates achieved after gradient, swim up and simple wash procedures

\begin{tabular}{llll}
\hline Procedure type & Number & No. biochem. pregnant & \% Biochem. pregnant \\
\hline Gradient Procedure & 684 & 109 & 16 \\
Swim Up Procedure & 261 & 54 & 21 \\
Simple Wash & 45 & 4 & 9 \\
Total Procedures & 990 & 167 & 17
\end{tabular}

Table 3 Comparison of clinical pregnancy rates and type of baby gender after gradient or swim up procedure

\begin{tabular}{llllllll}
\hline Procedure & $\begin{array}{l}\text { Clinically } \\
\text { pregnant (\#) }\end{array}$ & $\begin{array}{l}\text { Clinically } \\
\text { pregnant (\%) }\end{array}$ & Delivered (\#) & Male born (\#) & $\begin{array}{l}\text { Male born } \\
(\%)\end{array}$ & $\begin{array}{l}\text { Female } \\
\text { born (\#) }\end{array}$ & $\begin{array}{l}\text { Female born } \\
(\%)\end{array}$ \\
\hline Gradient & 84 & 12.3 & 61 & 29 & 48 & 32 & 52 \\
Swim Up & 43 & 16.3 & 26 & 13 & 50 & 13 & 50 \\
Total & 127 & 13 & 87 & 42 & 48 & 45 & 52 \\
\hline
\end{tabular}

\section{Discussion}

Since many centuries the ability to choose the sex of one's child has been desired by couples. Depending on the existing knowledge of the time, suggestions were made to preselect the baby gender. Aristotle advised sexual intercourse in Northern wind to get a boy and in Southern wind to get a girl. Millot proposed that it depends on the side, the female lies at the time of ejaculation: always a boy when she is on the right side and always a girl on the left side. ${ }^{9}$ In 1973, use of albumin gradients for separation of X- and Y- bearing spermatozoa by Ericson gained high popularity, ${ }^{4}$ however, conflicting results were obtained by other studies. Over the years, the knowledge evolved as new technologies became available. Before the advent of PGT, the most common laboratory procedures used for sperm separation were gradient filtration, swim up or swim down, albumin gradient filtration and sperm sorting by flow-cytometry followed by IUI or ICSI. However, based on the accuracy, only flow-cytometry and PGT are reliable methods. ${ }^{10}$ Due to relatively higher cost, the patients are still being advised to use density gradient or swim up followed by IUI. The purpose of this study was to investigate whether semen processing by density gradients or swim up procedure produce baby of desired gender.

Results of this study indicate that $40 / 80 \%$ gradients and swim up procedures failed to deviate child sex ratio from natural 50:50. To our knowledge, this is the first study comparing gender outcome of 945 IUI cycles when sperm were processed by gradients or swim up. The preconception or preimplantation sex selection by reliable procedure will help avoid abortion. The patients who wish to avoid abortion but who desire to select the sex of their offspring should consider either flow-cytometry for sperm separation or PGT for embryo sex selection. Although the accuracy of the flow-cytometry sperm separation is not $100 \%$, human sperm sorted by flow cytometry can significantly increase the likelihood that a child so conceived will be of a desired sex. ${ }^{11}$
In this era of advanced molecular genetic testing and due to the unreliable outcome of gradients, swim up and other similar gender preselection procedures, precise sex-selection prior to conception is desired to completely avoid physical and emotional trauma and ethical issues associated with induced abortion. ${ }^{12}$ Thus, among the techniques currently available, PGT of IVF/ICSI embryos followed by transfer of embryo (s) of desired gender is the best choice. The advances in preconception and preimplantation technologies make it clinically possible for parents to select the sex of their future child ${ }^{10}$ with almost $100 \%$ accuracy.

\section{Conclusion}

The data of this study indicate that sperm separation by gradients and swim-up does not change gender ratio from the natural 50:50, therefore, should not be recommended to patients for the purpose of preselection of baby gender.

\section{Data availability}

The original data used to support the findings of this study are available from the corresponding author upon request.

\section{Funding statement}

The authors (Except Ruaa Laqwer) are employee of Thuriah Medical Centre. Ruaa Laqwer volunteered for data organization and analysis. No research funding was available.

\section{Acknowledgments}

Authors are thankful to Thuriah Medical Center for providing data and resources to complete this work.

\section{Conflicts of interest}

The authors declare that there is no conflict of interest regarding the publication of this paper. 


\section{References}

1. Van Balen F. Attitudes towards sex selection in the Western world Prenatal Diagnosis. 2006;26(7):614-618.

2. Baheri S, Saki G, Mohammadi Asl J, et al. Assessment of the separation of X-and Y-chromosome bearing sperms of fertile men by albumin gradients using real time PCR. Jentashapir Journal of Health Research. 2016.

3. Jain T, Missmer SA, Gupta RS, et al. Preimplantation sex selection demand and preferences in an infertility population. Fertil Steril. 2005;83(3):649-658.

4. Ericsson RJ, Langevin CN, Nishino M. Isolation of fractions rich in human Y sperm. Nature. 1973;246(5433):421-424.

5. Check JH, Shanis BS, Cooper SO, et al. Male sex preselection: swimup technique and insemination of women after ovulation induction. Arch Androl. 1989;23(2):165-166.

6. Kaneko S, Yamaguchi J, Kobayashi T, et al. Separation of human X-and Y-bearing sperm using Percoll density gradient centrifugation. Fertil Steril. 1983;40(5):661-665.
7. Choavaratana R. Effect of abstinence duration on sperm sex ratio. Siriraj Medical Journal. 2014;66(4):103-112.

8. Dondorp W, De Wert G, Pennings G, et al. ESHRE Task Force on ethics and Law 20: sex selection for non-medical reasons. Hum Reprod. 2013;28(6):1448-1454.

9. Cohen J. Gender selection: is there a European view? J Assist Reprod Genet. 2002;19(9):417-419.

10. Ethics Committee of the American Society for Reproductive Medicine. Use of reproductive technology for sex selection for nonmedical reasons. Fertil Steril. 2015;103(6):1418-1422.

11. Karabinus DS, Marazzo DP, Stern HJ, et al. The effectiveness of flow cytometric sorting of human sperm (MicroSort $\left.{ }^{\circledR}\right)$ for influencing a child's sex. Reprod Biol Endocrinol. 2014;12(1):106.

12. Heng BC. Regulated family balancing by equalizing the sex-ratio of gender-selected births. J Assist Reprod Genet. 2006;23(7):319-320. 